\title{
1 Idiosynchrony: From shared responses to individual differences during
}

\section{2 naturalistic neuroimaging}

4 Authors: Emily S. Finn ${ }^{1 *}$, Enrico Glerean ${ }^{2}$, Arman Y. Khojandi ${ }^{1}$, Dylan Nielson ${ }^{3}$, Peter J.

5 Molfese $^{1}$, Daniel A. Handwerker ${ }^{1}$, Peter A. Bandettini ${ }^{1}$

7 'Section on Functional Imaging Methods, Laboratory of Brain and Cognition, National Institute 8 of Mental Health, Bethesda, Md. USA

$9 \quad{ }^{2}$ Department of Neuroscience and Biomedical Engineering, Aalto University, Espoo, Finland

$10 \quad{ }^{3}$ Mood Brain \& Development Unit, National Institute of Mental Health, Bethesda, Md. USA

$11 *$ Corresponding author: emily.finn@nih.gov

13 Abstract

14 Two ongoing movements in human cognitive neuroscience have researchers shifting focus from

15 group-level inferences to characterizing single subjects, and complementing tightly controlled

16 tasks with rich, dynamic paradigms such as movies and stories. Yet relatively little work

17 combines these two, perhaps because traditional analysis approaches for naturalistic imaging

18 data are geared toward detecting shared responses rather than between-subject variability. Here,

19 we review recent work using naturalistic stimuli to study individual differences, and advance a

20 framework for detecting structure in idiosyncratic patterns of brain activity, or "idiosynchrony".

21 Specifically, we outline the emerging technique of inter-subject representational similarity

22 analysis (IS-RSA), including its theoretical motivation and an empirical demonstration of how it

23 recovers brain-behavior relationships during movie watching using data from the Human

24 Connectome Project. We also consider how stimulus choice may affect the individual signal and

25 discuss areas for future research. We argue that naturalistic neuroimaging paradigms have the

26 potential to reveal meaningful individual differences above and beyond those observed during

27 traditional tasks or at rest.

29 Keywords: individual differences, naturalistic, inter-subject correlation, fMRI, behavior, 30 representational similarity analysis 


\section{$1 \quad$ Highlights}

2 - Review literature using naturalistic paradigms to study individual differences

3 - Discuss potential utility of idiosyncratic time-locked responses ("idiosynchrony")

4 - Outline technique of inter-subject representational similarity analysis (IS-RSA)

5 - Apply IS-RSA to reveal brain-behavior relationships during movie watching

6 - Consider role of stimulus selection and other directions for future work 


\section{1. Introduction}

2 At present, there are two exciting movements afoot in cognitive neuroscience. First, the field is

3 shifting focus from the group to the individual: instead of combining data across subjects to draw

4 population-level inferences, studies are isolating brain function in single subjects and how it

5 relates to behavioral phenotypes (Bartolomeo et al., 2017; Dubois and Adolphs, 2016; Seghier and Price, 2018). Second, researchers are embracing the complexity of so-called "naturalistic"

7 stimuli-e.g., film clips, spoken narratives — as experimental paradigms, to complement and

8 extend the tightly controlled, parametric tasks that form the pillars of classical psychology work

9 (Sonkusare et al., 2019).

11 While each of these movements has brought promising discoveries on its own, studies at the 12 intersection - i.e., that use naturalistic stimuli to study individual differences - are relatively

13 uncommon. Why might this be? Naturalistic stimuli evoke patterns of brain activity that are, by

14 and large, highly consistent across subjects (Hasson et al., 2010; Hasson et al., 2004), and

15 typically, naturalistic imaging data are collected and analyzed in ways geared toward detecting

16 similarities between subjects rather than differences (Nastase et al., 2019). Adapting naturalistic

17 tasks to study individual differences and brain-behavior relationships raises several challenges,

18 demanding new approaches to both experimental design and data analysis. The potential payoff,

19 however, is high. Naturalistic tasks offer a "happy medium" between the extremes of highly

20 controlled cognitive tasks, which often lack ecological validity, and resting-state acquisitions,

21 which are entirely unconstrained, making them vulnerable to confounds and difficult to interpret

22 (Vanderwal et al., 2018). They allow experimenters to probe intermingled signals throughout the

23 hierarchy of neural systems - from low-level sensory processing up to social cognition — using

24 data from a single acquisition, and while the brain is engaged in activities more similar to

25 everyday life. Thus, they may offer more "bang for the buck" than either rest or traditional tasks

26 for characterizing spatiotemporal patterns of brain activity in individual subjects.

28 Here, our goals are threefold. In the first section, we briefly describe the inter-subject correlation

29 (ISC) family of approaches to analyzing naturalistic imaging data, which exploit the time-locked

30 nature of the task across subjects to isolate brain activity driven by the stimulus (Hasson et al.,

31 2004; Nastase et al., 2019). Also in this section, we review existing literature using ISC to study 
1 individual differences. (For comprehensive reviews on naturalistic tasks and inter-subject

2 correlation more generally, we refer the reader to (Sonkusare et al., 2019) and (Nastase et al.,

3 2019), respectively. Our focus here is specifically on how these intersect with the study of

4 individual differences.) In the second section, we advance a framework for studying how

5 individual differences shape brain responses during naturalistic stimulation, a phenomenon we

6 call "idiosynchrony". We focus specifically on the emerging technique of inter-subject

7 representational similarity analysis (IS-RSA), which adapts ISC to highlight stimulus-driven

8 responses that are idiosyncratic rather than shared. We present the theory behind IS-RSA, then

9 apply it to data from the Human Connectome Project to demonstrate how brain responses during

10 movie viewing share structure with behavioral traits, including working memory and personality.

11 In the final section, we outline directions for future work, including how idiosynchrony might be

12 further adapted to serve as predictive model, and how we might choose stimuli to amplify

13 behaviorally relevant individual signals.

\section{2. Inter-Subject Correlation: Stimulus-locked, fewer assumptions}

16 Because naturalistic paradigms sit somewhere between resting state and traditional tasks,

17 researchers have at their disposal a wide variety of analysis approaches. The presence of a

18 stimulus permits classic techniques for detecting activation to specific features of the stimulus,

19 such as the general linear model (GLM). At the same time, thanks to their continuous, non-

20 parametric nature, these paradigms also lend themselves to approaches developed primarily for

21 rest - for example, functional connectivity, which considers correlations in signal fluctuations

22 between regions, rather than magnitude of activation in any single region.

24 We place these approaches in a landscape with two major axes: how time-locked to the stimulus

25 they are, and how many assumptions they require. Functional connectivity (FC) - especially

26 static FC, which collapses across the whole time series - demands relatively few assumptions,

27 but is impossible to map back onto the stimulus, and therefore fails to separate stimulus-driven

28 from stimulus-independent sources of neural activity. Dynamic FC gets closer to a time-resolved

29 signal, but in most cases the need for windows of a minimum length makes it difficult to map

30 connectivity fluctuations to specific events within the stimulus, and resulting signals still contain

31 a mixture of stimulus-related and stimulus-unrelated variation within each subject. 
2 GLM-based analyses do capitalize on the "ground truth" of a stimulus with known timing, but in

3 doing so make two critical assumptions: one, that the experimenter knows which features of the

4 stimulus are important for driving brain activity, and two, that they have modeled these features

5 accurately. Non-optimal assumptions at either step will impair sensitivity.

7 The inter-subject correlation (ISC) family of approaches (Hasson et al., 2004; Nastase et al., 8 2019; Simony et al., 2016), developed specifically for naturalistic paradigms, maximize 9 sensitivity to stimulus-driven activity with fewer assumptions. These approaches use one 10 subject's brain activity as a model for a second subject's brain activity, reasoning that as long as

11 two subjects receive the same input at the same time, any shared variance must be due to

12 stimulus processing. Unlike FC, ISC isolates stimulus-driven signal and is interpretable with

13 respect to events in the stimulus itself. And unlike GLM-based approaches, even if the

14 experimenter doesn't know — or can't model — the most important features of the stimulus, as

15 long as there is some consistent signal across subjects, ISC will recover it (Pajula et al., 2012).

16 Thus, ISC is a powerful, data-driven technique for detecting shared responses, anticipated or 17 otherwise.

\subsection{Adapting ISC to an individual-differences framework}

20 The classic formulation of ISC assumes that the signal observed at each voxel $x$ and timepoint $t$ 21 reflects a mixture of three components. Loosely following the notation of Nastase et al. (2019),

22 these components are:

Thus, for a given subject $i$ the response in voxel $x$ at timepoint $t$ can be described as follows: $i d$, a stimulus-evoked response that is idiosyncratic to each subject $\varepsilon$, a noise component (which may reflect both neural activity that is unrelated to the stimulus, i.e., mind-wandering, as well as noise from non-neural physiological and scanner sources)

$$
\mathrm{x}_{i}(\mathrm{t})=\mathrm{c}(\mathrm{t})+\mathrm{id}_{i}(\mathrm{t})+\varepsilon_{i}(\mathrm{t})
$$


2 In this formulation, it is not possible to distinguish $i d$ from $\varepsilon$, unless subjects are exposed to the 3 same stimulus multiple times. Even then, results must be interpreted with caution, because

4 repetition can change how a stimulus is processed. Another approach is to anchor id to some 5 other known information about each subject, like a behavioral score $(b)$, and search for structure 6 in these responses across subjects:

13 Notice that now the $i d$ term has lost its subject subscript, since we are assuming that there is

14 some canonical response associated with a given behavior, and each subject's behavioral score

15 acts as a sort of bias term governing to what degree a subject expresses that response.

17 This framework let us distinguish stimulus-related responses in individual subjects from

18 stimulus-unrelated noise, and does so in a way that facilitates interpretation, since we are linking

19 idiosyncratic responses to a known behavioral measure. Generally, we might predict that the

20 influence of individual differences on $x(t)$ - that is, the ratio of $i d$ to $c$ - grows as one moves up

21 the cortical processing hierarchy, such that the shared, behavior-independent signal dominates in

22 unimodal cortex, while the idiosyncratic, behavior-dependent signal becomes stronger in higher-

23 order association cortex. This may explain why ISC is traditionally high in primary visual and

24 auditory cortex, and drops off (but doesn't necessarily disappear completely) in areas of

25 prefrontal cortex, for example: it is not the case that these regions are not responding to the

26 stimulus, but rather that they respond with different spatiotemporal signatures across subjects

27 (Chang et al., 2018; Hasson et al., 2010). Some of this variance might be explained by trait-level 28 phenotypes. 
1 Despite the traditional focus on shared responses, in recent years, researchers have begun to investigate how ISC varies with both state-level factors related to either the stimulus or experimental instructions, and - more relevant for our purposes - with trait-like factors intrinsic

4 to the subjects themselves.

Several studies have shown that ISC is sensitive to features of the stimulus (Dmochowski et al.,

7 2014; Hasson et al., 2010; Nummenmaa et al., 2014; Schmälzle et al., 2015). Other studies have 8 kept the stimulus constant and used behavioral priming to show that ISC is sensitive to explicit manipulations of attention or prior beliefs about a stimulus (Cooper et al., 2011; Lahnakoski et al., 2014; Yeshurun et al., 2017). While informative, these studies do not examine why different

11 individuals often spontaneously - i.e., with no explicit priming - show different neural or

12 behavioral responses to the same stimulus.

14 A handful of studies have reported spontaneous individual differences in time-locked activity to 15 a stimulus that relate to behavioral appraisal of the stimulus. Using emotional clips, comedy

16 videos, and moral dilemma scenarios, respectively, Nummenmaa et al. (2012), Jaaskelainen et al. 17 (2016) and Tei et al. (2019) found that ISC in certain brain regions correlated with similarity of 18 post-hoc behavioral ratings (of dynamic valence/arousal, humor, and moral conflict) across pairs 19 of subjects. Using an abstract video of animated shapes and an audio story, respectively, Nguyen 20 et al. (2019) and Saalasti et al. (2019) found that subject pairs with higher ISC in certain cortical 21 regions during the stimulus ultimately shared more similar interpretations of the stimulus. These 22 studies provide a fascinating window into how individual differences in brain activity during 23 stimulus exposure relate to subsequent differences in reactions and interpretations. But because 24 both brain responses and behavior are tied to the specific stimulus at hand, the extent to which 25 these state-like differences reflect trait-like predispositions-i.e., intrinsic individual 26 characteristics - remains unclear.

28 Recent studies take these analyses one step further and link idiosyncratic responses during 29 naturalistic stimulation to stable, trait-like factors. Finn et al. (2018) report that individuals with 30 higher trait paranoia show stronger ISC in cortical regions involved in social processing during 31 an ambiguous social narrative. Bacha-Trams et al. (2018) report that individual differences in 
1 cognitive style (i.e., holistic versus analytical thinking) relate to strength of ISC in several

2 cortical regions during a drama movie. In a sample of college-age males, Chen et al (2019) report

3 that variations in sociosexual desire preferences are linked to variations in brain activity in

4 reward, default-mode and mentalizing networks, and self-control preferences to variations in

5 executive and salience networks, during erotic (but not neutral) films. In a sample of children

6 and adolescents spanning ages 7-21 years, Gruskin et al. (2019) report that patterns and severity

7 of depression symptoms are linked to brain responses during an emotionally evocative animated

8 clip, but only in adolescents, suggesting that these idiosyncratic responses emerge over the

9 course of development. Finally, in a study of individuals with dyslexia, ISC calculated from

10 MEG envelope time series correlated with similarity in phonological processing, technical

11 reading, and working memory (Thiede et al., 2019). Encouragingly, these studies show that there

12 is meaningful - i.e., behaviorally relevant - structure in idiosyncratic responses to naturalistic

13 stimuli.

15 Other studies have addressed variability by dichotomizing subjects into diagnostic groups.

16 Several studies have reported differences in ISC during naturalistic paradigms between healthy

17 controls and populations with mental illnesses and disorders-most commonly, autism (Bolton et

18 al., 2018; Byrge et al., 2015; Hasson et al., 2009; Salmi et al., 2013), but also depression (Guo et

19 al., 2015) and first-episode psychosis (Mäntylä et al., 2018; Yang et al., 2019). In general, these

20 studies report reduced cross-subject synchrony in the patient group, an effect that sometimes

21 scales with symptom severity (Guo et al., 2015; Salmi et al., 2013).

23 Group contrasts between patients and controls can be useful to assess population-level

24 differences in broad strokes. But rather than distinct canonical responses for each group, patients

25 are typically characterized by increased heterogeneity of responses (Bolton et al., 2018). This

26 suggests that a better framework would approach the question as an individual-differences

27 problem. Indeed, Byrge et al.'s post-hoc analyses revealed that their ISC effect was driven

28 entirely by five individuals from the ASD group with particularly idiosyncratic responses that

29 could not be easily explained by other factors (i.e., data quality, symptom severity); when these

30 five individuals were removed, the ASD and neurotypical groups were indistinguishable.

31 Interestingly, Hasson et al. (2009) reported that while responses subjects with autism were more 
1 variable, these idiosyncratic responses were reliable within single individuals across repeat

2 presentations of the same stimulus, suggesting a trait-like component.

4 Rather than a dichotomy between health and disease, most mental illnesses are likely better 5 conceptualized as the extreme end of a phenotypic spectrum (Cuthbert and Insel, 2013; Insel et

6 al., 2010). Instead of stratifying subjects into patients and controls, we can improve sensitivity by

7 using continuous measures - task performance, symptom severity, genetic load — as our

8 independent variables (Finn and Constable, 2016). To then take advantage of the richness of

9 information embedded in these continuous spaces, we need analysis frameworks that can

10 appropriately handle the intricate interdependencies of dyads rather than individuals. Recent

11 work has proposed using multilevel modeling and other expanded statistical formulations, some

12 of which offer the ability to include subject-level covariates into ISC analyses (Chen et al.,

13 2017). In the next section, we outline another promising approach, used by several of the studies

14 cited above, that is flexible, intuitive and can be applied to detect relationships between any type

15 of brain and behavioral data.

\section{4. Inter-subject Representational Similarity Analysis: Theory}

18 By definition, inter-subject correlation cannot be calculated for a single subject. How, then, can

19 we relate ISC, which operates at the level of subject pairs, to traits and behaviors, which operate

20 at the level of single subjects?

22 We can compute a similarity score between pairs of individuals using other types of data, for 23 example self-report questionnaires, demographics, task performance, clinical assessments, or 24 genotypes, among others. The intuition is that individuals who are more similar in behavior

25 should also be more similar in their neural responses. We can operationalize this by computing 26 two subject-by-subject distance matrices: one for the brain data (using, for example, ISC), and

27 one for the behavioral data. We can then compare the geometry of these two matrices by 


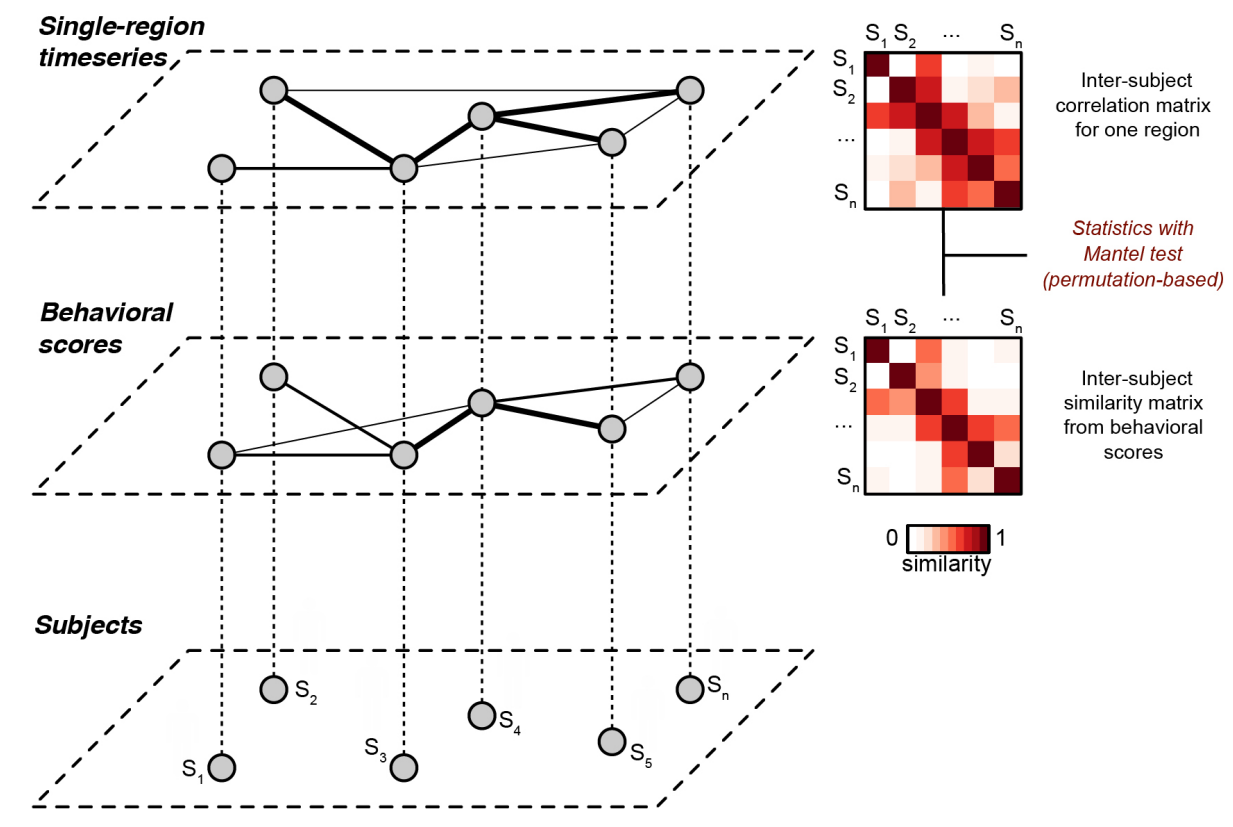

Figure 1. Schematic of inter-subject representational similarity analysis. Each subject (bottom layer) is associated with a behavioral score (middle layer) and a pattern of brain activity (top layer; in this case, a time series from a given brain region during naturalistic stimulation). In ISRSA, we construct pairwise (i.e, subject-by-subject) similarity matrices for the behavioral data and the brain data, then compare these matrices using a Mantel test. Thus, we can leverage inter-subject analysis methods such as ISC to detect shared structure between brain data and behavioral data. This figure is a modified version of Fig. 1 in Glerean et al. (2016).

correlating them, a procedure known as a Mantel test (Mantel, 1967) or representational similarity analysis (Kriegeskorte et al., 2008). We and others call this inter-subject representational similarity analysis (Chen et al., 2019; van Baar et al., 2019). The advantage of comparing similarity matrices over a typical first-level analysis is that instead of directly linking two physically different quantities like brain data and behavior, we use a second-order isomorphism to compare the geometry of brain data with the geometry of behavioral data (Figure 1) (Kriegeskorte and Kievit, 2013).

9 This sounds straightforward enough. But one critical question is, how do we measure behavioral 10 similarity? In choosing a distance metric, particularly when our behavior is one-dimensional

11 (e.g., age (Moraczewski et al., 2018; Richardson et al., 2018), a trait score, accuracy on a

12 cognitive task), we imbue our analysis with some fundamental assumptions about the structure of

13 the brain-behavioral representational similarity that affect the ultimate results and how we

14 interpret them. To get a feel for some potential structures, imagine arranging the rows and 
1 columns of the ISC matrix such that subjects are ordered by their behavioral score. What would

2 we expect the resulting matrix to look like?

3

4 If we use Euclidean distance or another relative distance metric, we implicitly assume that

5 subjects with closer scores should look more similar to one another, regardless of where they fall

6 on the scale. In other words, for a behavior that is measured on a scale from 0 to 100 , a pair of

7 subjects scoring 0 and 1 should be just as similar to one another as a pair of subjects scoring 99

8 and 100 (since in both cases, the Euclidean distance is 1). We call this the Nearest Neighbors

9 model, since it assumes that a subject should always look most similar to his or her immediate

10 neighbors, regardless of their absolute position on the scale (Figure 2a). 
$\operatorname{abs}(\mathrm{i}-\mathrm{j})$

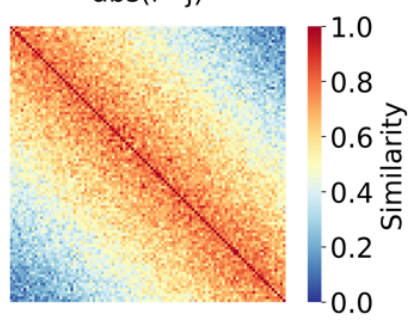

b

mean $(\mathrm{i}, \mathrm{j})$
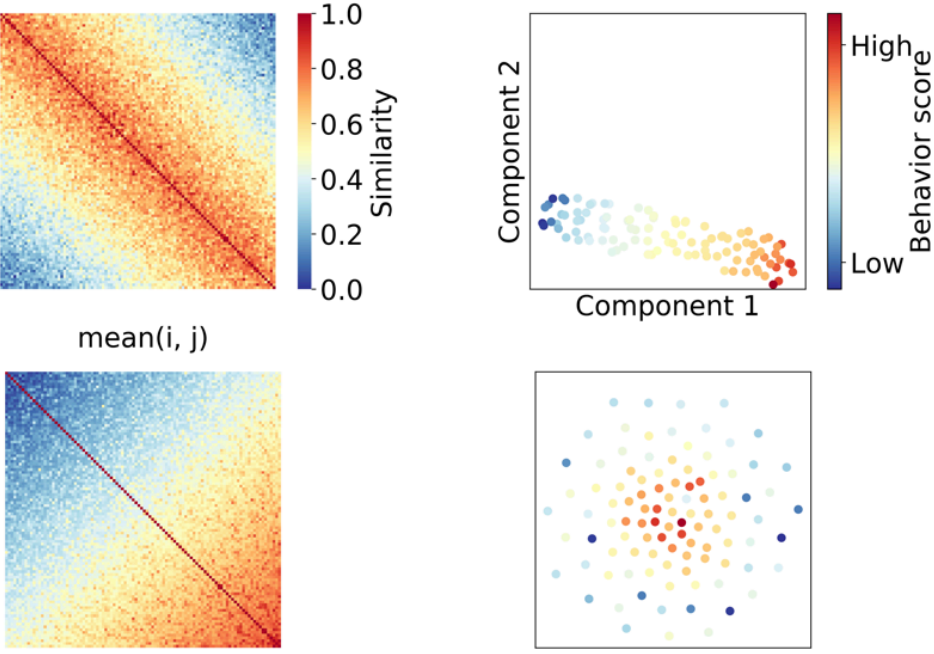

7

Linear

"Nearest Neighbors"

C

$\min (i, j)$
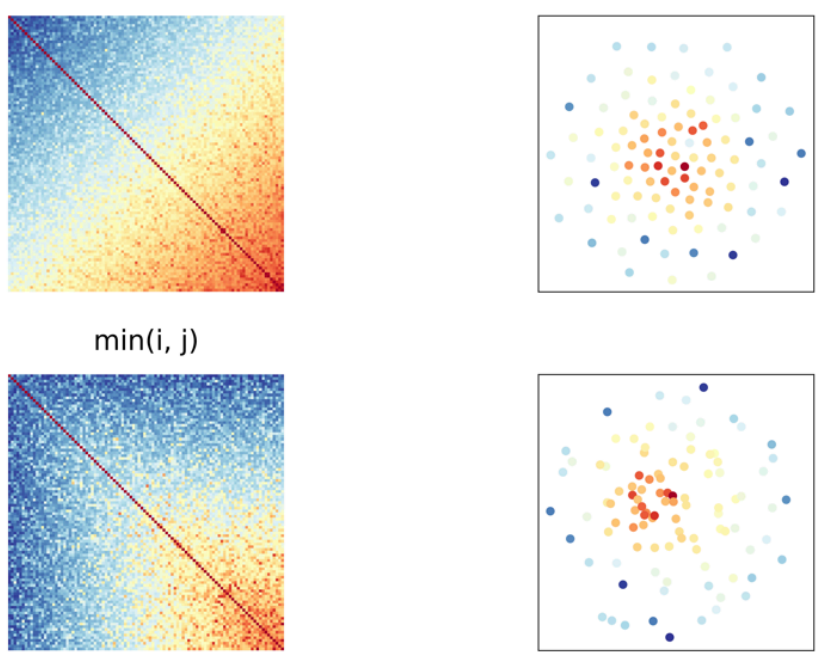

Non-linear

"Anna Karenina"

d $\quad \operatorname{abs}(\mathrm{i}-\mathrm{j}) *$ mean $(\mathrm{i}, \mathrm{j})$
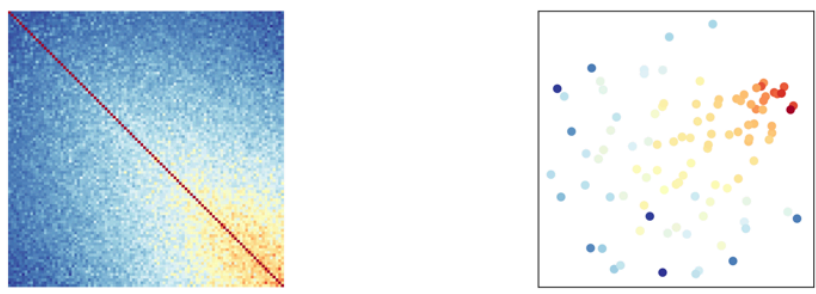

Figure 2. Simulated potential structures for brain-behavior representational similarity matrices. For each row a-d, the left panel depicts a simulated pairwise brain similarity matrix in which subjects are ordered along both rows $i$ and columns $j$ by their behavioral score (from low to high), and each cell $\{i, j\}$ reflects the correlation between subjects $i$ and $j$ of the timeseries of a given brain region (pairwise inter-subject correlation). The right panel depicts a two-dimensional embedding of the corresponding distance matrix (i.e., 1 - similarity matrix) using t-SNE (t-Distributed Stochastic Neighbor Embedding), in which each dot represents a subject, and subjects are colored according to their behavioral score. Several potential structures are possible, including linear and non-linear relationships.

2 The NN model may be appropriate for certain behaviors, but we could imagine an equally if not

3 more plausible scenario: that similarity between subjects increases or decreases as one moves up

4 or down the scale, in an absolute rather than relative sense. For example, perhaps high-scoring

5 subjects are more similar to other high scorers, while low-scoring subjects are less similar both to

6 high scorers and other low scorers. In other words, brain responses cluster together for subjects

7 at one end of the behavioral spectrum, while variability increases as one moves toward the 
1 opposite end of the spectrum. We call this the Anna Karenina (or AnnaK) model, after the

2 famous opening line of Leo Tolstoy's novel, which reads "All happy families are alike; each

3 unhappy family is unhappy in its own way" (or, in this context, "all high [low] scorers are alike;

4 each low [high] scorer is different in his or her own way"). In this case, Euclidean distance

5 would not be the most appropriate choice. Instead, we would want to model similarity using a

6 nonlinear metric that reflects absolute position-for example, mean: $(i+j) / 2$, minimum: $\min (i$,

$7 j$ ), or the product of the mean and minimum (Figure 2b-d).

9 In the case of traits and behaviors that consist of a vector of responses per subject (e.g., self-

10 report questionnaires) rather than a single scalar number (e.g., age), we have the option to

11 calculate item-wise similarity using any number of potential distance metrics-for example,

12 correlation, Euclidean distance, cosine distance and many others. In this case, we are assuming

13 that it is the pattern of individual responses, and not the composite score, that should determine

14 the inter-subject similarity structure. This approach is likely best suited to assessments consisting

15 of unique items that are not interchangeable - personality questionnaires, for example ${ }^{1}$. As a

16 general heuristic, quantitative assessments - those with clear "better" and "worse" ends of the

17 scale - are likely more suited to distance models based on single composite scores, while

18 qualitative scales could be suited to either composite or item-wise models. We can also imagine

19 scenarios where both models are theoretically appropriate, and each might capture different

20 effects. For example, questionnaires assessing symptom type and severity might show some

21 effects that scale with overall score (Anna Karenina model), and others that scale with item-wise

22 similarity (nearest neighbors) - and each of these effects might be present in different brain

23 regions.

\section{5. Inter-subject RSA: Application to HCP data}

26 To investigate if and how brain similarity reflects behavioral similarity during naturalistic

27 stimulation, and more specifically, how the choice of distance model affects results, we applied

\footnotetext{
${ }^{1}$ Since most cognitive performance tests consist of more than one item, we could in theory calculate item-wise distance on these tests as well. But because trials in these tests are generally interchangeable, it is more straightforward and interpretable to consider the similarity of two subjects' composite score rather than the similarity of their performance on individual trials (unless one has a specific hypothesis about learning rates, attention fluctuations, or other effects with a dynamic component).
} 
1 inter-subject RSA to an empirical dataset from the Human Connectome Project. Subjects

2 engaged in a movie-watching paradigm during high-resolution (voxel size $=1.6 \mathrm{~mm}^{3}, \mathrm{TR}=1 \mathrm{~s}$ )

3 functional MRI scanning at 7 Tesla. The sample used here ( $\mathrm{n}=184$, from 93 unique families)

4 reflects all available data for this paradigm. Details of data acquisition and basic preprocessing

5 are published elsewhere (Glasser et al., 2013; Van Essen et al., 2012; Vu et al., 2017). Each

6 subject watched four 15-minute movie runs; data from the first run (MOVIE1_7T_AP) are used

7 here. This run comprised five video clips presented in a fixed order. Four clips were from

8 independent films and documentaries, and one was a montage of brief (1.5s) moving scenes.

10 Each subject also completed a battery of self-report and behavioral measures outside the scanner

11 (Barch et al., 2013). We focused on two trait-level measures from the cognitive and emotional

12 domains, respectively: working memory (as measured by a list-sorting task) and personality (as

13 measured by the NEO Five-Factor Inventory). We chose these two traits because we

14 hypothesized that the structure of brain-behavior similarity would manifest differently for each

15 one, and therefore that each trait would be best modeled by a different distance function in IS-

16 RSA, as detailed below.

18 The primary outcome measure from the list-sorting task is a measure of working memory span,

19 or how many items can be accurately stored and manipulated in working memory at one time.

20 Because it yields a single scalar score per subject, behavioral similarity can be computed

21 according to either a nearest-neighbors or AnnaK model. The NEO-FFI consists of 60 items, and

22 subsets of these are summed to yield scores for five dimensions: agreeableness, extraversion,

23 conscientiousness, neuroticism, and openness. Therefore, for personality, we can compute

24 behavioral similarity in any of three ways: (1) AnnaK for each of the five traits (to test the

25 prediction, e.g., that people who are more agreeable share similar patterns of brain activity, while

26 people that are less agreeable show more variability); (2) nearest-neighbors for each of the five

27 traits (people that score more similarly on agreeableness look more similar, regardless of whether

28 they score high or low); or (3) nearest-neighbors for all 60 item-wise responses (people who fill

29 out the questionnaire in more similar ways, regardless of their summary trait scores, will show

30 more similar brain activity). We tested all possible models for each behavior (two for working

31 memory and three for personality). We hypothesized that working memory would be best 
1 captured by an AnnaK model in most brain regions. We did not have strong hypotheses about the

2 best model for personality, as any of the above scenarios were plausible a priori.

4 Because we might expect both behavioral phenotypes and brain activity to be more similar

5 between siblings - and especially twins - due to any number of genetic and environmental

6 influences, we avoided performing IS-RSA on related individuals. We split the dataset into two

7 cohorts of unrelated subjects ( $\mathrm{n}=93$ and $\mathrm{n}=89$, respectively), which had the benefit of giving us

8 a natural replication sample to help guard against false positives. We performed all analyses on

9 each cohort separately, and only relationships that appeared in both cohorts were considered

10 "significant".

12 Beginning with the FIX-denoised data, for each subject, we extracted activity timecourses from

13 every node in a 268-node functional parcellation (Shen atlas; (Shen et al., 2013)) by averaging

14 signal across all voxels for each volume. (Because activity is expected to be smooth across

15 neighboring voxels, this step reduces the dimensionality of the data, thus avoiding the

16 computational cost of a voxelwise analysis.) For each node $n$ and each subject pair $\{i, j\}$ within a

17 cohort, brain similarity was calculated as the Pearson correlation (ISC) across the whole run.

18 Behavioral similarity was calculated according to either an NN model $(i-j$; Figure $2 \mathrm{a})$ or an

19 AnnaK model (mean $(i, j)$; Figure 2b). 
Working Memory
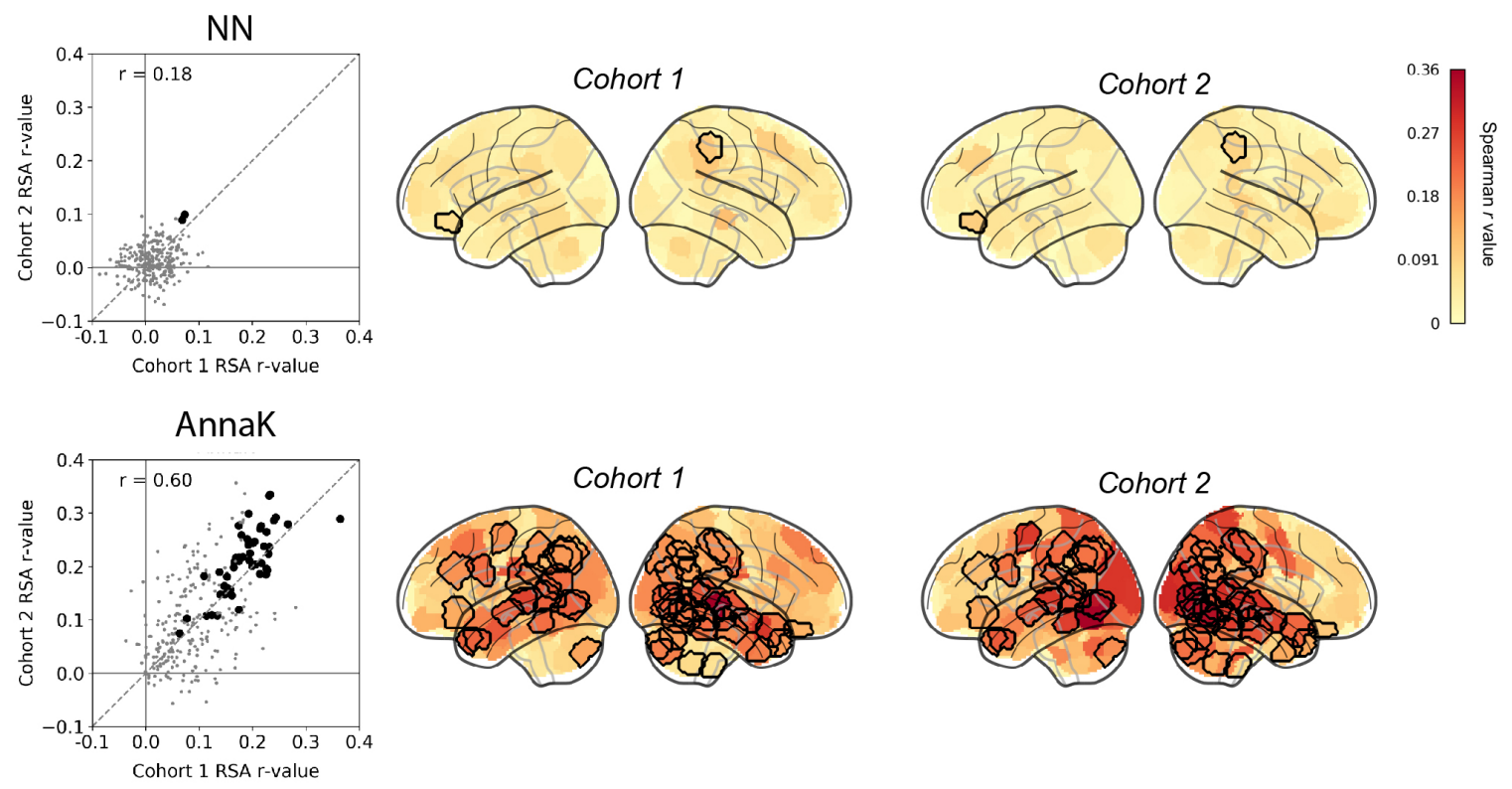

Figure 3. Inter-subject RSA applied to HCP data: Working Memory. Do pairs of subjects that score more similarly on a test of working memory (HCP: ListSort) also show stronger ISC in certain brain regions during naturalistic viewing? Two models for behavioral similarity are tested: a nearest-neighbor model (top row; cf. Figure 2a) where the behavioral similarity matrix is constructed as $|\mathrm{i}-\mathrm{j}|$, and an "Anna Karenina" model (bottom row, cf. Figure 2b) where the behavioral similarity matrix is constructed as mean(i,j). In the scatter plots, each dot represents one node in the Shen atlas (268 total), plotted according to its representational similarity (Spearman correlation between brain similarity and behavioral similarity matrix) in cohort 1 ( $x$-axis) versus its representational similarity in cohort 2 (y-axis). Large black dots are nodes that show significant representational similarity $(p<0.05)$ after permutation testing (no. permutations $=10,000)$ in both cohorts. Dashed lines along the diagonal represent the identity line (not the regression line), to facilitate visual inspection of replicability-ideally, the RSA values would be similarly distributed in cohort 1 and cohort 2, and the correlation between the two cohorts would be close to 1. Glass brains show nodes colored by is-RSA value. Nodes outlined in black show significant representational similarity in both cohorts (corresponding to the large black dots in the scatterplots).

1 For working memory, as we hypothesized, representational similarity between brain and

2 behavior was best captured by the AnnaK model (Figure 3, bottom row). High-scoring subjects

3 looked similar to other high scorers across much of the brain, while low-scoring subjects had

4 more idiosyncratic responses - in other words, they looked less similar to both the high scorers

5 and other low scorers. The AnnaK model fit the data better than the nearest-neighbors model in

6 that it yielded stronger effect sizes (compare distributions of RSA values between the two scatter

7 plots in Figure 3) that were more replicable across the two subject cohorts $\left(\mathrm{r}_{\text {cohort } 1 \text {, cohort } 2}=0.60\right.$

8 for the AnnaK model versus 0.18 for the NN model). The AnnaK model captured significant

9 relationships between brain and behavioral similarity in 53 nodes across parietal, temporal, 


\section{two nodes.}

a. Personality: Itemwise
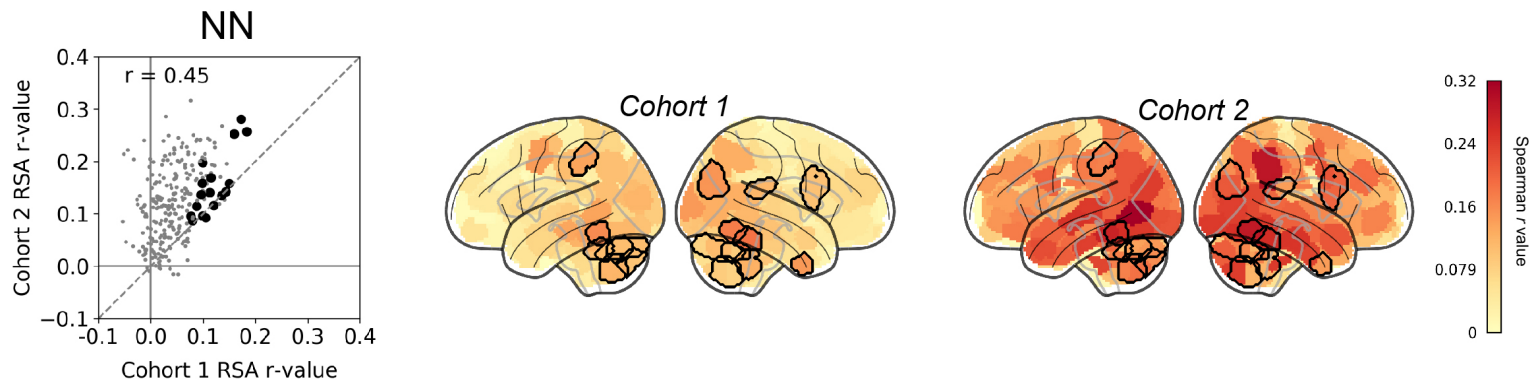

b. Personality: Trait scores

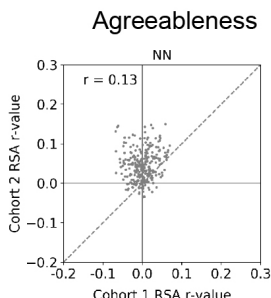

Cohort 1 RSA r-value

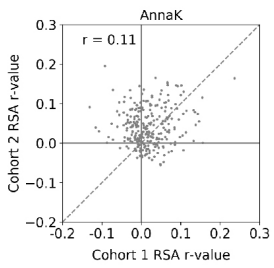

(n.s.)
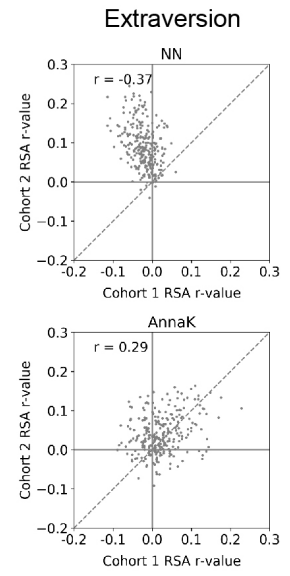

(n.s.)

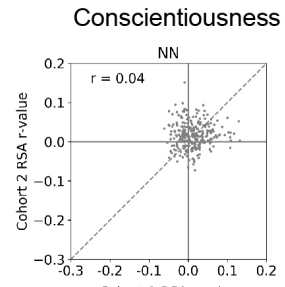

Cohort 1 RSA r-value
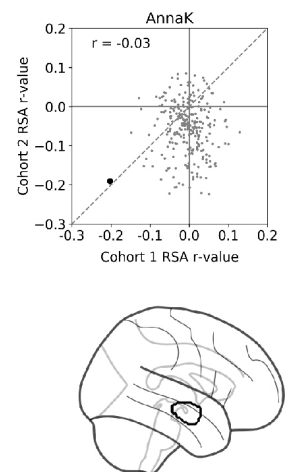

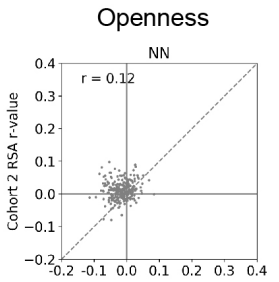

Cohort 1 RSA r-value
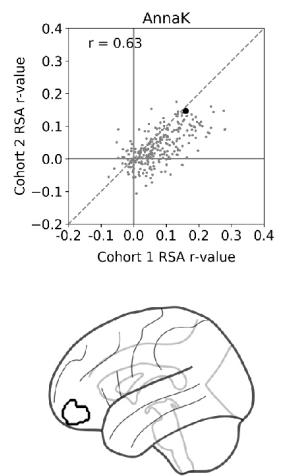

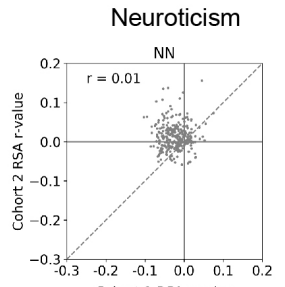

Cohort 1 RSA r-value

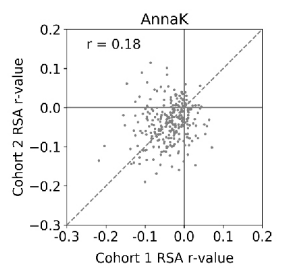

(n.s.)

Figure 4. Inter-subject RSA applied to HCP data: Personality. Do pairs of subjects with more similar personalities (as measured with the Five-Factor Inventory) also show stronger ISC in certain brain regions during naturalistic viewing? a) IS$R S A$, where personality similarity is calculated as the Pearson correlation between item-wise responses of each pair of subjects ("NN-itemwise"). b) IS-RSA where personality similarity is calculated based on summary scores for each of the five traits. For each trait, two models for behavioral similarity are tested: a nearest-neighbor model (top graph in each column; cf. Figure 2a) where the behavioral similarity matrix is constructed as $|\mathrm{i}-\mathrm{j}|$, and an "Anna Karenina" model (bottom graph in each column, cf. Figure $2 b$ ) where the behavioral similarity matrix is constructed as mean $(\mathrm{i}, \mathrm{j})$. In all scatter plots, each dot represents one node in the Shen atlas (268 total), plotted according to its representational similarity (Spearman correlation between brain similarity and behavioral similarity matrix) in cohort 1 ( $x$-axis) versus its representational similarity in cohort 2 (y-axis). Large black dots are nodes that show significant representational similarity $(p<0.05)$ after permutation testing in both cohorts. Dashed lines along the diagonal represent the identity line (not the regression line), to facilitate visual inspection of replicability-ideally, the RSA values would be similarly distributed in cohort 1 and cohort 2, and the correlation between the two cohorts would be close to 1. Nodes outlined in black on show significant representational similarity in both cohorts (same as those in the scatterplots). Traits with no corresponding glass brain figure did not have any nodes that were significant in both cohorts ("n.s."). 
1 For personality, results were more complicated. Using trait summary scores, the nearest-neighbor

2 model based on trait summary scores did not yield any significant nodes across any of the five

3 dimensions (Figure 4b, top scatter plots), and the AnnaK model yielded only two significant

4 nodes across all five dimensions (Figure 4b, bottom scatter plots): one in the left middle

5 temporal lobe that showed higher ISC among pairs of subjects scoring low on conscientiousness

6 (Figure 4b, glass brain), and one in the orbitofrontal cortex that showed higher ISC among pairs

7 of subjects scoring high on openness (Figure 4b, glass brain). However, the nearest-neighbor

8 model based on item-wise responses captured several (16) nodes across the brain (Figure 4a),

9 including fusiform, right inferior frontal gyrus, and several nodes in the cerebellum.

10 Interestingly, this suggests that brain responses during naturalistic stimulation may depend less

11 on overall levels of certain personality traits, and more on personality "fingerprints" captured by

12 specific patterns of item-wise responses to the personality questionnaire.

14 In sum, we observed that different traits and behaviors are best modeled by different distance

15 functions in different brain regions. These results raise several questions to be addressed in

16 future work. For example, which model(s) perform best on other traits and behaviors? Can we

17 use dynamic analyses (Glerean et al., 2012; Simony et al., 2016) to reveal particular time

18 windows where the brain-behavior signal is strongest? Do these windows relate to known

19 features of the stimulus? For now, we can conclude that IS-RSA is a promising framework to

20 detect brain-behavior relationships during naturalistic imaging, and that choosing the appropriate

21 model not only improves sensitivity, but also offers the flexibility to test multiple hypotheses

22 with fundamentally different interpretations.

\section{6. Inter-subject RSA: Future directions}

25 Extending IS-RSA to a predictive framework

26 So far, we have discussed inter-subject RSA as a way to relate brain similarity to behavioral

27 similarity in a fixed set of subjects. Eventually, however, we would like to build models that can

28 generate predictions about never-before-seen individuals (Bzdok and Ioannidis, 2019; Gabrieli et

29 al., 2015). How might we extend IS-RSA to a predictive framework? 
1 In many cases, we might want to build a decoding model, which uses a subject's pattern of brain

2 activity during naturalistic stimulation to generate a predicted behavioral score for that subject.

3 The AnnaK models predict a monotonic relationship between a subject's behavioral score and

4 their mean (or median) ISC value with all other subjects. Therefore, a simple model might

5 parameterize this relationship in a set of training subjects, then calculate the mean ISC for a held-

6 out subject with all subjects in the training set and input this value into the model to generate a

7 predicted behavior. Alternatively, one could build a model based on a "canonical" timecourse

8 calculated from high (or low) scorers in the training set and generate a predicted score for a held-

9 out subject based on their distance from this canonical timecourse.

11 The NN models do not predict any relationship between behavior and overall mean or median

12 ISC value, but rather between behavior and ISC with specific partners. To translate this to a

13 predictive model, we could use a $k$-nearest neighbors framework that would predict a held-out

14 subject's behavioral score as a weighted average of scores from the $k$ subjects in the training set

15 with whom they show the highest ISC.

17 In either case, certain brain regions would likely show a stronger relationship between ISC and

18 behavior than other regions. Feature selection could be applied to uncover the brain regions

19 where similarity is most strongly related to behavioral similarity, to improve model performance.

21 Alternatively, we could invert this framework to build encoding models, which, given a stimulus

22 and a behavioral score, predict a subject's timecourse of brain activity. While this may prove

23 more difficult, a successful model would provide a mapping for how an intrinsic trait and a

24 complex stimulus interact to produce activity in a given brain region (Naselaris et al., 2011), a

25 powerful step forward in understanding how complex stimuli are filtered through an individual

26 lens.

Unsupervised IS-RSA

29 As we amass larger datasets that include naturalistic neuroimaging, it may become possible to

30 perform unsupervised analyses. In other words, instead of using known behavioral scores to pre-

31 label the data, we could cluster inter-subject brain similarity matrices to detect natural categories 
1 or continua in a data-driven fashion, then see if these relate to present or future behavioral

2 outcomes (Cerliani et al., 2017). In this way, we may be able to leverage naturalistic

3 neuroimaging to organize individuals along axes that are more biologically valid than current

4 diagnostic and self-report measures, potentially shedding new light on how individual variability

5 is reflected in nuanced brain function.

\section{More sophisticated temporal techniques}

8 Thus far we have been using inter-subject correlation, or simple Pearson correlation between two

9 subjects' timeseries, as our primary measure of brain similarity, largely because it is

10 straightforward to compute, visualize, and interpret. However, in theory we could calculate a

11 brain similarity matrix (cf. Figure 1) based on any type of information we might extract from

12 single subjects' neuroimaging data. For example, we could use functional connectivity (Glerean

13 et al., 2016), or compare subjects' temporal trajectories over the course of a stimulus using low-

14 dimensional topological embeddings (e.g., (Gonzalez-Castillo et al., 2019; Saggar et al., 2018)),

15 latent state discovery (Chang et al., 2018), or projection into a higher-order space using recurrent

16 neural networks (Venkatesh et al., 2019). Furthermore, the brain and behavioral inter-subject

17 similarity matrices (cf. Figure 1) can be also interpreted as networks. This approach allows for

18 more robust measures of similarities between two networks without the strong dependence on

19 the actual link weights and even allows for missing data points between subject pairs (Peixoto,

20 2015; Schmidt and Morup, 2013). These approaches may prove even more powerful for

21 uncovering brain-behavior relationships during naturalistic imaging, though potentially at the

22 expense of some interpretability.

\section{Stimulus selection: How much synchrony is enough?}

25 Thus far, we have discussed how to optimize sensitivity to stimulus-evoked individual

26 differences from an analysis perspective. What about from an acquisition perspective? While

27 naturalistic imaging experiments are growing in popularity, currently, there is no principled way

28 to choose stimuli. Common wisdom is that for studying shared responses, we should choose

29 something maximally engaging, to "drive" as much of the brain as possible. Indeed, previous

30 work has shown that features of the stimulus affect ISC levels: more rhetorically powerful

31 speeches (Schmälzle et al., 2015), emotionally arousing narratives (Nummenmaa et al., 2014), 
1 and highly rated television programs (Dmochowski et al., 2014) all evoke higher synchrony than

2 their less engaging counterparts.

4 But, when the goal of a study is specifically to investigate individual differences, considerations

5 for choosing a stimulus may be different. In the theoretical limit, a stimulus that evoked perfect

6 synchrony across subjects would be useless for studying individual differences — since there

7 would be no neural variability to relate to behavioral variability (Hedge et al., 2018). Practically,

8 however, we are quite far from that theoretical limit, since individual BOLD responses are

9 "noisy" both in terms of uninteresting variability (scanner noise, non-neural BOLD signals,

10 stimulus-unrelated neural activity) and the stimulus-driven idiosyncratic responses that constitute

11 the "signal" of interest here.

13 Is there a "sweet spot" where a stimulus evokes enough synchrony to build a successful cross-

14 subject model, but not enough to saturate the individual signals of interest? To test their

15 hypothesis that social network proximity predicts increasingly similar neural responses to

16 movies, Parkinson et al. (2018) chose videos that might differentially appeal to those with

17 different tastes (reasoning that friends would be more likely to have similar tastes; e.g., styles of

18 humor, opinions on controversial topics). A handful of studies have created bespoke stimuli that

19 were ambiguous by design, such that different individuals might arrive at different

20 interpretations of the same material. Finn et al (2018) created a narrative describing a complex

21 social scenario that seemed highly suspicious or nefarious to some individuals, but less so to

22 others; Nguyen et al (2018) created a Heider-Simmel-esque video (Heider and Simmel, 1944)

23 depicting an interaction among animated shapes in which the relationships between the shapes

24 were open to interpretation. These studies found that individuals who were more similar on

25 either trait-level (i.e., intrinsic) or state-level (i.e., stimulus-driven) measures, respectively,

26 showed increased inter-subject correlation during stimulus presentation in regions of higher-

27 order association cortices, especially those linked to social cognition.

29 None of these studies, however, directly assessed how the level of ambiguity or so-called "taste30 dependence" of a stimulus affects its utility for drawing out meaningful individual signal. If the

31 goal is to study idiosyncratic responses, how much synchrony is optimal? If we imagine plotting 
1 stimuli by the strength of the ISCs they evoke versus their sensitivity to individual differences,

2 several potential scenarios emerge (Figure 5. ). In theory, idiosyncratic signals might be quickly

3 saturated by a powerful stimulus, such that the optimal stimulus would evoke only minimally

4 correlated responses when averaged across all subjects, leaving room for specific subject pairs to

5 be more or less correlated with one another according to variations in behavior (Scenario 1).

6 Alternatively, the optimal level of average synchrony could be higher, such that sensitivity to

7 individual differences benefits from a stronger foundational shared response at the group level

8 (Scenario 2). Another possibility is that the theoretical "saturation point" is much higher, such

9 that stimuli that evoke very strong responses are the ones that are most sensitive to individual

10 differences (Scenario 3). This third scenario would be consistent with the observation that stimuli

11 and task states that make subjects look more similar to one another can actually boost signal-to-

12 noise for individual differences, since even though these states reduce overall cross-subject

13 variation, the remaining variation is presumably more stable and trait-like (Finn et al., 2017;

14 Vanderwal et al., 2017).

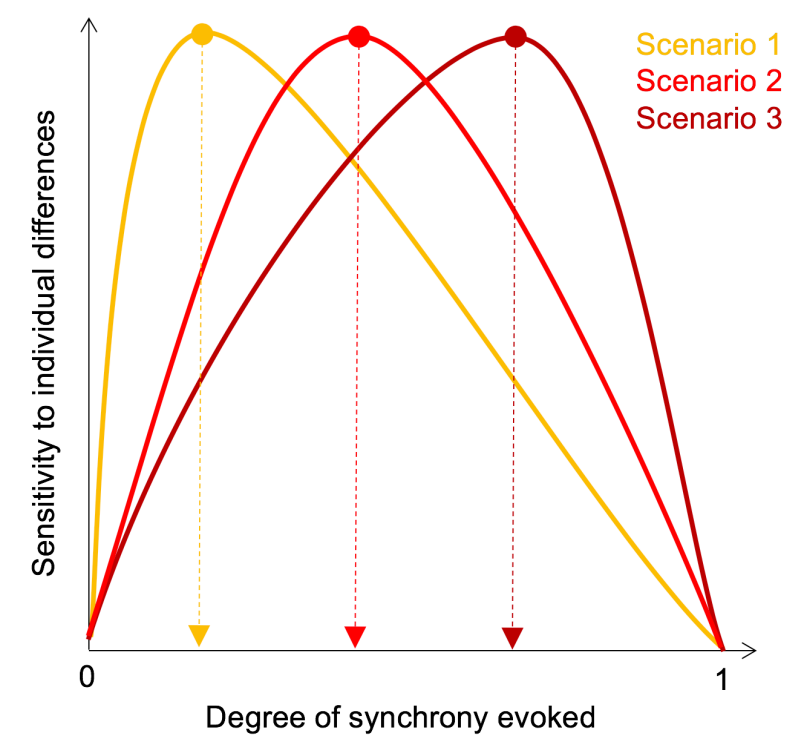

Figure 5. Theoretical stimulus tuning curves for sensitivity to individual differences. In the upper limit, as the degree of cross-subject synchrony evoked by a stimulus approaches 1 , that stimulus will lose sensitivity to individual differences, since there will be no brain variability left to relate to behavioral variability. However, in the lower limit, if a stimulus evokes no correlation across subject, there will be no meaningful structure in brain similarity to relate to behavioral similarity. Therefore, the optimal tuning curve likely follow an inverted-U shape. Determining the peak of this curve-in other words, the optimal degree of synchrony for extracting meaningful individual differences in a certain behavioral domain-should be a goal for future work.
Understanding how stimulus choice affects sensitivity to individual differences will be an important area for future research, especially as large-scale data collection efforts begin to incorporate naturalistic scans into their protocols (Alexander et al., 2017; Van Essen et al., 2012). We believe acquiring naturalistic scans should be encouraged for several reasons: beyond improving subject compliance (and therefore data quality) compared to resting-state scans (Greene et al., 2018; Huijbers et al., 2017; Vanderwal et al., 2015), naturalistic paradigms yield data that can be mined in any number of ways, expanding their potential to generate insights into a variety of open questions in human 
1 neuroscience. Yet, to the extent that stimulus choice affects the individual signals we observe, it

2 will behoove us to be as principled as possible in choosing stimuli.

4 Of course, there may not be a single "best" stimulus for studying individual differences; rather,

5 the most appropriate stimulus may depend on the specific behavior(s) of interest. For example, a

6 threatening or suspenseful stimulus - e.g., the opening scene in a horror film - might evince

7 neural responses that share structure with trait anxiety, as compared to one depicting positive

8 emotion-e.g., the happily-ever-after final scene of a romantic comedy. Conversely, the positive

9 stimulus might yield better predictions of trait anhedonia. At the same time, both of these stimuli

10 may yield better predictions of either trait than a neutral stimulus with little to no emotional

11 content. Testing these hypotheses will require datasets with a range of both stimuli and

12 behavioral measures per subject. However, there is already some supporting evidence: for

13 example, differences between controls and patients with melancholic depression are more

14 pronounced during a negative film clip than a positive one (Guo et al., 2015).

\section{8. Limitations}

17 Despite the many clear advantages and — we believe — great potential of using naturalistic

18 paradigms to study individual differences, there are several outstanding challenges. For one, the

19 test-retest validity of these paradigms is unclear. Repetition is known to alter neural processing,

20 and this is likely especially true for emotionally evocative and memorable stimuli. Thus,

21 naturalistic paradigms that rely on a single stimulus may be less appropriate for longitudinal

22 studies, since it would be near impossible to disentangle within-subject changes from repetition

23 effects.

25 There are computational challenges associated with using inter-subject approaches, since

26 pairwise techniques mean that the number of observations increases as $n^{2}$ rather than $n$.

27 Especially in the case of ISFC, reducing dimensionality using pre-defined atlases, and/or

28 selecting regions of interest based on a priori hypotheses or a first-pass analysis of which regions

29 surpass some minimal threshold for response consistency (cf. Figure 5. ), may help in this

30 regard. 
1 Another challenge, albeit not one unique to naturalistic paradigms, is understanding which traits

2 and behaviors we should target. Predicting a performance-based or self-report measure acquired

3 close in time to the imaging data itself, which is the goal of the vast majority of the current

4 literature on brain-behavior relationships, is an important proof-of-concept, but ultimately the

5 imaging data will always be simply a noisier version of whatever "ground truth" we are trying to

6 predict. Determining the added value of neuroimaging will require using baseline brain

7 responses to naturalistic stimuli to predict follow-up measures such as learning rates (Cantlon

8 and $\mathrm{Li}, 2013)$, illness trajectory, or response to intervention.

\section{Concluding remarks}

11 Here, we have advanced an emerging framework for studying individual differences during

12 naturalistic neuroimaging, a phenomenon we call "idiosynchrony". Inter-subject representational

13 similarity analysis (IS-RSA) combines the time-locked nature of the stimulus with known

14 phenotypic information to move from shared responses to activity in individual subjects that is

15 idiosyncratic, yet structured and interpretable. Unlike traditional approaches that rely on explicit

16 models of the task, inter-subject approaches promise to capture as much nuance and variance of

17 the evoked activity as possible. And, unlike functional connectivity approaches that treat

18 naturalistic neuroimaging data akin to rest, inter-subject approaches afford near certainty that the

19 observed signals are both neural in origin and driven by the stimulus. Above and beyond a boost

20 in signal-to-noise, these paradigms open up exciting opportunities to link individual patterns of

21 brain activity to specific events within the stimulus that unfold over various timescales, from its

22 low-level sensory properties up to high-level narrative features that may evoke different

23 memories, associations and emotions for each individual. Linking naturalistic patterns of brain

24 activity to trait- and state-related variability across subjects will deepen our understanding of

25 how individual brains give rise to individual behaviors, and may eventually help empower

26 imaging-based tools for real-world applications. 


\section{Acknowledgements}

2 This work was supported by the National Institutes of Health (grant K99MH120257 to E.S.F.

3 and the ZIAMH002783 to P.A.B.). Portions of this study used the computational capabilities of

4 the NIH HPC Biowulf cluster (hpc.nih.gov). Data were provided in part by the Human

5 Connectome Project, WU-Minn Consortium (Principal Investigators: David Van Essen and

6 Kamil Ugurbil; 1U54MH091657) funded by the 16 NIH Institutes and Centers that support the

7 NIH Blueprint for Neuroscience Research; and by the McDonnell Center for Systems

8 Neuroscience at Washington University.

10 Data and code availability

11 Raw data for the empirical results presented here come from the Human Connectome Project

12 (http://www.humanconnectomeproject.org/). Code for all IS-RSA analyses — both simulations

13 (cf. Figure 2) and empirical application to HCP data (cf. Figure 3, Figure 4) - can be found in

14 the following Github repository: https://github.com/esfinn/intersubj_rsa, which also contains the

15 processed HCP data (nodewise time series) that formed the input to the empirical analyses. 
Alexander, L.M., Escalera, J., Ai, L., Andreotti, C., Febre, K., Mangone, A., Vega-Potler, N., Langer, N., Alexander, A., Kovacs, M., et al. (2017). An open resource for transdiagnostic research in pediatric mental health and learning disorders. Scientific Data 4, 170181.

Bacha-Trams, M., Alexandrov, Y.I., Broman, E., Glerean, E., Kauppila, M., Kauttonen, J., Ryyppö, E., Sams, M., and Jääskeläinen, I.P. (2018). A drama movie activates brains of holistic and analytical thinkers differentially. Soc Cogn Affect Neurosci 13, 1293-1304.

Barch, D.M., Burgess, G.C., Harms, M.P., Petersen, S.E., Schlaggar, B.L., Corbetta, M., Glasser, M.F., Curtiss, S., Dixit, S., Feldt, C., et al. (2013). Function in the human connectome: TaskfMRI and individual differences in behavior. Neuroimage 80, 169-189.

Bartolomeo, P., Seidel Malkinson, T., and de Vito, S. (2017). Botallo's error, or the quandaries of the universality assumption. Cortex $86,176-185$.

14 Bolton, T.A.W., Jochaut, D., Giraud, A.L., and Ville, D.V.D. (2018). Brain dynamics in ASD during movie-watching show idiosyncratic functional integration and segregation. Hum Brain Mapp 39, 2391-2404.

17 Byrge, L., Dubois, J., Tyszka, J.M., Adolphs, R., and Kennedy, D.P. (2015). Idiosyncratic Brain Activation Patterns Are Associated with Poor Social Comprehension in Autism. J Neurosci 35, 5837-5850.

Bzdok, D., and Ioannidis, J.P.A. (2019). Exploration, Inference, and Prediction in Neuroscience and Biomedicine. Trends Neurosci 42, 251-262.

22 Cantlon, J.F., and Li, R. (2013). Neural activity during natural viewing of Sesame Street statistically predicts test scores in early childhood. PLoS Biol 11, e1001462.

24 Cerliani, L., Thomas, R.M., Aquino, D., Contarino, V., and Bizzi, A. (2017). Disentangling subgroups of participants recruiting shared as well as different brain regions for the execution of the verb generation task: A data-driven fMRI study. Cortex 86, 247-259.

27 Chang, L.J., Jolly, E., Cheong, J.H., Rapuano, K., Greenstein, N., Chen, P.-H.A., and Manning, 28 J.R. (2018). Endogenous variation in ventromedial prefrontal cortex state dynamics during 29 naturalistic viewing reflects affective experience. bioRxiv, 487892.

30 Chen, G., Taylor, P.A., Shin, Y.-W., Reynolds, R.C., and Cox, R.W. (2017). Untangling the 31 relatedness among correlations, Part II: Inter-subject correlation group analysis through linear 32 mixed-effects modeling. Neuroimage 147, 825-840.

33 Chen, P.-H.A., Jolly, E., Cheong, J.H., and Chang, L.J. (2019). Inter-subject representational 34 similarity analysis reveals individual variations in affective experience when watching erotic 35 movies. bioRxiv, 726570. 
1 Cooper, E.A., Hasson, U., and Small, S.L. (2011). Interpretation-mediated changes in neural

2 activity during language comprehension. Neuroimage 55, 1314-1323.

3 Cuthbert, B.N., and Insel, T.R. (2013). Toward the future of psychiatric diagnosis: the seven

4 pillars of RDoC. BMC Med 11, 126.

5 Dmochowski, J.P., Bezdek, M.A., Abelson, B.P., Johnson, J.S., Schumacher, E.H., and Parra,

6 L.C. (2014). Audience preferences are predicted by temporal reliability of neural processing.

7 Nature communications 5, 4567.

8 Dubois, J., and Adolphs, R. (2016). Building a Science of Individual Differences from fMRI.

9 Trends in Cognitive Sciences 20, 425-443.

10 Finn, E.S., and Constable, R.T. (2016). Individual variation in functional brain connectivity:

11 implications for personalized approaches to psychiatric disease. Dialogues Clin Neurosci 18,

$12 \quad 277-287$.

13 Finn, E.S., Corlett, P.R., Chen, G., Bandettini, P.A., and Constable, R.T. (2018). Trait paranoia 14 shapes inter-subject synchrony in brain activity during an ambiguous social narrative. Nature

15 Communications 9, 2043.

16 Finn, E.S., Scheinost, D., Finn, D.M., Shen, X., Papademetris, X., and Constable, R.T. (2017).

17 Can brain state be manipulated to emphasize individual differences in functional connectivity?

18 Neuroimage 160, 140-151.

19 Gabrieli, John D.E., Ghosh, Satrajit S., and Whitfield-Gabrieli, S. (2015). Prediction as a

20 Humanitarian and Pragmatic Contribution from Human Cognitive Neuroscience. Neuron 85, 11 -

2126.

22 Glasser, M.F., Sotiropoulos, S.N., Wilson, J.A., Coalson, T.S., Fischl, B., Andersson, J.L., Xu,

23 J., Jbabdi, S., Webster, M., and Polimeni, J.R. (2013). The minimal preprocessing pipelines for

24 the Human Connectome Project. Neuroimage 80, 105-124.

25 Glerean, E., Pan, R.K., Salmi, J., Kujala, R., Lahnakoski, J.M., Roine, U., Nummenmaa, L.,

26 Leppämäki, S., Nieminen-von Wendt, T., and Tani, P. (2016). Reorganization of functionally

27 connected brain subnetworks in high-functioning autism. Hum Brain Mapp 37, 1066-1079.

28 Glerean, E., Salmi, J., Lahnakoski, J.M., Jääskeläinen, I.P., and Sams, M. (2012). Functional

29 Magnetic Resonance Imaging Phase Synchronization as a Measure of Dynamic Functional

30 Connectivity. Brain Connectivity 2, 91-101.

31 Gonzalez-Castillo, J., Caballero-Gaudes, C., Topolski, N., Handwerker, D., Pereira, F., and 32 Bandettini, P. (2019). Imaging the spontaneous flow of thought: Distinct periods of cognition 33 contribute to dynamic functional connectivity during rest. bioRxiv, 527804.

34 Greene, D.J., Koller, J.M., Hampton, J.M., Wesevich, V., Van, A.N., Nguyen, A.L., Hoyt, C.R., 35 McIntyre, L., Earl, E.A., Klein, R.L., et al. (2018). Behavioral interventions for reducing head 36 motion during MRI scans in children. Neuroimage 171, 234-245. 
1 Gruskin, D.C., Rosenberg, M.D., and Holmes, A.J. (2019). Relationships between depressive

2 symptoms and brain responses during emotional movie viewing emerge in adolescence. bioRxiv, 3542720.

4 Guo, C.C., Nguyen, V.T., Hyett, M.P., Parker, G.B., and Breakspear, M.J. (2015). Out-of-sync: 5 disrupted neural activity in emotional circuitry during film viewing in melancholic depression.

6 Sci Rep 5, 11605.

7 Hasson, U., Avidan, G., Gelbard, H., Vallines, I., Harel, M., Minshew, N., and Behrmann, M.

8 (2009). Shared and idiosyncratic cortical activation patterns in autism revealed under continuous

9 real-life viewing conditions. Autism Research 2, 220-231.

10 Hasson, U., Malach, R., and Heeger, D.J. (2010). Reliability of cortical activity during natural

11 stimulation. Trends in cognitive sciences $14,40-48$.

12 Hasson, U., Nir, Y., Levy, I., Fuhrmann, G., and Malach, R. (2004). Intersubject Synchronization 13 of Cortical Activity During Natural Vision. Science 303, 1634-1640.

14 Hedge, C., Powell, G., and Sumner, P. (2018). The reliability paradox: Why robust cognitive 15 tasks do not produce reliable individual differences. Behav Res Methods 50, 1166-1186.

16 Heider, F., and Simmel, M. (1944). An experimental study of apparent behavior. The American 17 journal of psychology 57, 243-259.

18 Huijbers, W., Van Dijk, K.R.A., Boenniger, M.M., Stirnberg, R., and Breteler, M.M.B. (2017). 19 Less head motion during MRI under task than resting-state conditions. Neuroimage 147, 11120120.

21 Insel, T., Cuthbert, B., Garvey, M., Heinssen, R., Pine, D.S., Quinn, K., Sanislow, C., and Wang, 22 P. (2010). Research domain criteria (RDoC): toward a new classification framework for research 23 on mental disorders. Am J Psychiatry 167, 748-751.

24 Jääskeläinen, I.P., Pajula, J., Tohka, J., Lee, H.-J., Kuo, W.-J., and Lin, F.-H. (2016). Brain

25 hemodynamic activity during viewing and re-viewing of comedy movies explained by

26 experienced humor. Sci Rep 6, 27741.

27 Kriegeskorte, N., and Kievit, R.A. (2013). Representational geometry: integrating cognition, 28 computation, and the brain. Trends in cognitive sciences 17, 401-412.

29 Kriegeskorte, N., Mur, M., and Bandettini, P. (2008). Representational similarity analysis 30 connecting the branches of systems neuroscience. Front Syst Neurosci 2.

31 Lahnakoski, J.M., Glerean, E., Jääskeläinen, I.P., Hyönä, J., Hari, R., Sams, M., and

32 Nummenmaa, L. (2014). Synchronous brain activity across individuals underlies shared

33 psychological perspectives. Neuroimage 100, 316-324.

34 Mantel, N. (1967). The detection of disease clustering and a generalized regression approach. 35 Cancer Res 27, 209-220. 
Mäntylä, T., Nummenmaa, L., Rikandi, E., Lindgren, M., Kieseppä, T., Hari, R., Suvisaari, J.,

2 and Raij, T.T. (2018). Aberrant Cortical Integration in First-Episode Psychosis During Natural

3 Audiovisual Processing. Biol Psychiatry 84, 655-664.

4 Moraczewski, D., Chen, G., and Redcay, E. (2018). Inter-subject synchrony as an index of

5 functional specialization in early childhood. Sci Rep 8, 2252.

6 Naselaris, T., Kay, K.N., Nishimoto, S., and Gallant, J.L. (2011). Encoding and decoding in

7 fMRI. Neuroimage 56, 400-410.

8 Nastase, S.A., Gazzola, V., Hasson, U., and Keysers, C. (2019). Measuring shared responses

9 across subjects using intersubject correlation. Soc Cogn Affect Neurosci 14, 667-685.

10 Nguyen, M., Vanderwal, T., and Hasson, U. (2019). Shared understanding of narratives is

11 correlated with shared neural responses. Neuroimage 184, 161-170.

12 Nummenmaa, L., Glerean, E., Viinikainen, M., Jääskeläinen, I.P., Hari, R., and Sams, M. (2012).

13 Emotions promote social interaction by synchronizing brain activity across individuals. Proc Natl

14 Acad Sci USA 109, 9599-9604.

15 Nummenmaa, L., Saarimäki, H., Glerean, E., Gotsopoulos, A., Jääskeläinen, I.P., Hari, R., and

16 Sams, M. (2014). Emotional speech synchronizes brains across listeners and engages large-scale

17 dynamic brain networks. Neuroimage 102, 498-509.

18 Pajula, J., Kauppi, J.-P., and Tohka, J. (2012). Inter-Subject Correlation in fMRI: Method

19 Validation against Stimulus-Model Based Analysis. PLoS One 7, e41196.

20 Parkinson, C., Kleinbaum, A.M., and Wheatley, T. (2018). Similar neural responses predict

21 friendship. Nature Communications 9, 332.

22 Peixoto, T.P. (2015). Model Selection and Hypothesis Testing for Large-Scale Network Models

23 with Overlapping Groups. Physical Review X 5, 011033.

24 Richardson, H., Lisandrelli, G., Riobueno-Naylor, A., and Saxe, R. (2018). Development of the 25 social brain from age three to twelve years. Nature Communications 9, 1027.

26 Saalasti, S., Alho, J., Bar, M., Glerean, E., Honkela, T., Kauppila, M., Sams, M., and

27 Jääskeläinen, I.P. (2019). Inferior parietal lobule and early visual areas support elicitation of

28 individualized meanings during narrative listening. Brain and Behavior 9, e01288.

29 Saggar, M., Sporns, O., Gonzalez-Castillo, J., Bandettini, P.A., Carlsson, G., Glover, G., and

30 Reiss, A.L. (2018). Towards a new approach to reveal dynamical organization of the brain using 31 topological data analysis. Nature Communications 9, 1399.

32 Salmi, J., Roine, U., Glerean, E., Lahnakoski, J., Nieminen-von Wendt, T., Tani, P., Leppämäki,

33 S., Nummenmaa, L., Jääskeläinen, I.P., Carlson, S., et al. (2013). The brains of high functioning

34 autistic individuals do not synchronize with those of others. NeuroImage: Clinical 3, 489-497. 
Schmälzle, R., Häcker, F.E.K., Honey, C.J., and Hasson, U. (2015). Engaged listeners: shared neural processing of powerful political speeches. Soc Cogn Affect Neurosci 10, 1137-1143.

3 Schmidt, M.N., and Morup, M. (2013). Nonparametric Bayesian modeling of complex networks:

4 an introduction. IEEE Signal Processing Magazine 30, 110-128.

5 Seghier, M.L., and Price, C.J. (2018). Interpreting and utilising intersubject variability in brain 6 function. Trends in cognitive sciences 22, 517-530.

7 Shen, X., Tokoglu, F., Papademetris, X., and Constable, R. (2013). Groupwise whole-brain 8 parcellation from resting-state fMRI data for network node identification. Neuroimage 82,403 9415.

10 Simony, E., Honey, C.J., Chen, J., Lositsky, O., Yeshurun, Y., Wiesel, A., and Hasson, U.

11 (2016). Dynamic reconfiguration of the default mode network during narrative comprehension.

12 Nature Communications 7, 12141.

13 Sonkusare, S., Breakspear, M., and Guo, C. (2019). Naturalistic Stimuli in Neuroscience:

14 Critically Acclaimed. Trends in cognitive sciences.

15 Tei, S., Kauppi, J.-P., Fujino, J., Jankowski, K.F., Kawada, R., Murai, T., and Takahashi, H.

16 (2019). Inter-subject correlation of temporoparietal junction activity is associated with conflict

17 patterns during flexible decision-making. Neurosci Res 144, 67-70.

18 Thiede, A., Glerean, E., Kujala, T., and Parkkonen, L. (2019). Atypical brain-to-brain

19 synchronization during listening to continuous natural speech in dyslexia. bioRxiv, 677674 .

20 van Baar, J.M., Chang, L.J., and Sanfey, A.G. (2019). The computational and neural substrates

21 of moral strategies in social decision-making. Nature Communications 10, 1483.

22 Van Essen, D.C., Ugurbil, K., Auerbach, E., Barch, D., Behrens, T.E.J., Bucholz, R., Chang, A., 23 Chen, L., Corbetta, M., Curtiss, S.W., et al. (2012). The Human Connectome Project: A data 24 acquisition perspective. Neuroimage 62, 2222-2231.

25 Vanderwal, T., Eilbott, J., and Castellanos, F.X. (2018). Movies in the magnet: Naturalistic

26 paradigms in developmental functional neuroimaging. Dev Cogn Neurosci.

27 Vanderwal, T., Eilbott, J., Finn, E.S., Craddock, R.C., Turnbull, A., and Castellanos, F.X.

28 (2017). Individual differences in functional connectivity during naturalistic viewing conditions.

29 Neuroimage 157, 521-530.

30 Vanderwal, T., Kelly, C., Eilbott, J., Mayes, L.C., and Castellanos, F.X. (2015). Inscapes: A 31 movie paradigm to improve compliance in functional magnetic resonance imaging. Neuroimage $32 \quad 122,222-232$.

33 Venkatesh, M., Jaja, J., and Pessoa, L. (2019). Brain dynamics and temporal trajectories during 34 task and naturalistic processing. Neuroimage 186, 410-423. 
1 Vu, A.T., Jamison, K., Glasser, M.F., Smith, S.M., Coalson, T., Moeller, S., Auerbach, E.J., 2 Ugurbil, K., and Yacoub, E. (2017). Tradeoffs in pushing the spatial resolution of fMRI for the

3 7T Human Connectome Project. Neuroimage 154, 23-32.

4 Yang, Z., Wu, J., Xu, L., Deng, Z., Tang, Y., Gao, J., Hu, Y., Zhang, Y., Qin, S., Li, C., et al. 5 (2019). Individualized psychiatric imaging based on inter-subject neural synchronization in 6 movie watching. Neuroimage, 116227.

7 Yeshurun, Y., Swanson, S., Simony, E., Chen, J., Lazaridi, C., Honey, C.J., and Hasson, U. 8 (2017). Same story, different story: the neural representation of interpretive frameworks. Psychol 9 Sci $28,307-319$.

10 